\title{
Enhancing the nonlinear optical response and nonlinear figure- of-merit for silicon nanowires by integrating 2D graphene oxide films
}

This paper was downloaded from TechRxiv (https://www.techrxiv.org).

\section{LICENSE}

CC BY 4.0

SUBMISSION DATE / POSTED DATE

02-08-2020 / 04-08-2020

\section{CITATION}

Moss, David (2020): Enhancing the nonlinear optical response and nonlinear figure-of-merit for silicon nanowires by integrating 2D graphene oxide films. TechRxiv. Preprint.

https://doi.org/10.36227/techrxiv.12751190.v1

$\mathrm{DOI}$

10.36227/techrxiv.12751190.v1 


\title{
Enhancing the nonlinear optical response and nonlinear figure-of- merit for silicon nanowires by integrating 2D graphene oxide films
}

\author{
Yuning Zhang, Jiayang Wu, Yunyi Yang, Yang Qu, Tania Moein, \\ Baohua Jia, and David J. Moss* \\ Centre for Micro-Photonics, Swinburne University of Technology, Hawthorn, Victoria 3122, Australia. \\ Email:dmoss@swin.edu.au
}

\begin{abstract}
We experimentally demonstrate enhanced self-phase modulation in silicon nanowire waveguides integrated with layered graphene oxide films. We achieve spectral broadening of optical pulses in the GO-silicon waveguide with a broadening factor up to 2.96 .
\end{abstract}

\section{Introduction}

Nonlinear integrated photonic devices offer powerful solutions to generate and process signals all-optically, with far superior processing speed to electronic devices as well as the added benefits of compact footprint, low power consumption, high stability, and the potential to reduce cost by mass production [1]. Although silicon has been a leading platform for integrated photonic devices, its strong two-photon absorption (TPA) at near-infrared wavelengths poses a fundamental limitation for the performance of nonlinear silicon photonic devices in the telecommunications band operating via the Kerr nonlinearity [1-3] and even other $3^{\text {rd }}$ order nonlinearities such as four-wave mixing (FWM) [4,5] and third harmonic generation (THG) [6-11]. Despite the fact that TPA can sometimes be used to advantage, [12-14] this has motivated the search for both alternate platforms [15-22] or highly nonlinear materials that can be integrated onto chips to overcome these limitations [23-29]. Our GO papers

Owing to its ease of preparation as well as the tunability of its material properties, GO has become a highly promising member of the two-dimensional (2D) material family [30-32]. Previously, we reported GO films with a giant Kerr nonlinear response about 4 orders of magnitude higher than that of silicon [30] and demonstrated enhanced four-wave mixing (FWM) in doped silica waveguides integrated with GO films [31]. In this paper, we demonstrate enhanced self-phase modulation (SPM) in silicon nanowire waveguides integrated with 2D layered GO films. We conformally integrate 1-3 layers of GO on silicon waveguides using a large-area, transfer-free, layer-by-layer GO coating method and perform SPM measurements with picosecond optical pulses. Owing to the strong light-matter interaction between the silicon waveguides and the highly nonlinear GO films, a significant increase in broadening of pulses is achieved for the hybrid waveguides as compared with the bare silicon waveguide, with a high broadening factor (BF) of 2.96 being achieved for the device with 2 layers of GO. These results confirm the effectiveness of introducing 2D layered GO films into integrated silicon photonic devices to improve the performance of nonlinear optical processes.

\section{Device fabrication and characterization}

Figure 1(a) shows a schematic of a conformally GO-coated silicon nanowire waveguide. The silicon waveguides with a cross section of $500 \mathrm{~nm} \times 220 \mathrm{~nm}$ were fabricated on a silicon-on-insulator (SOI) wafer using 248-nm deep ultraviolet photolithography followed by an inductively coupled plasma etching process. Inverse taper couplers were employed to couple light into and out of the devices with lensed fibers. A 1.5- $\mu \mathrm{m}$ thick silica layer was deposited by plasma enhanced chemical vapor deposition as an upper cladding layer. Finally, a window with a length of $2.2 \mathrm{~mm}$ was opened down to the buried oxide layer to allow the introduction of GO films. The GO film was introduced onto the silicon chip by a solution-based method that yielded transfer-free, layer-by-layer GO film deposition [31, 32]. This method allows conformally coating GO films onto the silicon waveguide, as shown by the scanning electron micrograph (SEM) in Fig. 1(b) and the Raman spectra in Fig. 1(c). Figure 1(d) presents the SEM image of a GO film
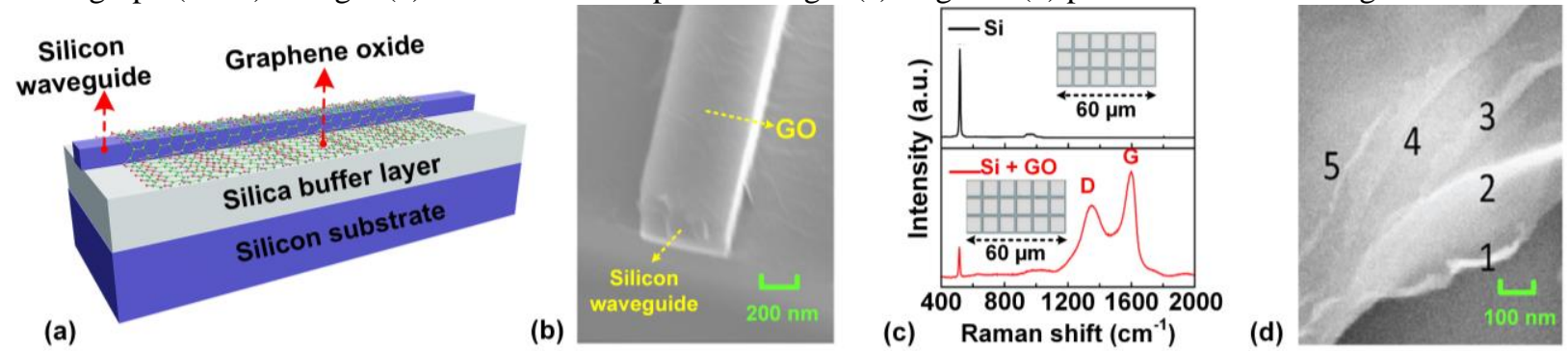

Fig. 1. (a) Schematic illustration of a GO-coated silicon nanowire waveguide. (b) Scanning electron micrograph (SEM) image of a silicon nanowire waveguide conformally coated with 1 layer of GO. (c) Raman spectra of an integrated chip without GO and with 2 layers of GO. Insets show the corresponding microscope images. (d) SEM image of layered GO film. The numbers refer to the number of layers for that part of the image. 
with up to 5 layers of GO, clearly showing the layered film structure. The GO film thickness shows a nearly linear relationship with the layer number, with a thickness of $\sim 2.06 \mathrm{~nm}$ on average for each layer. Fig. 2(a) depicts the insertion loss of the hybrid waveguides with 1-3 layers of GO measured with a low-power $(0 \mathrm{dBm}) \mathrm{CW}$ light at 1550 $\mathrm{nm}$. The GO layers only affected the propagation (not coupling) loss, which is shown in Fig. 2(b). The propagation loss of the bare waveguide and the waveguide with a monolayer of GO was $\sim 0.42 \mathrm{~dB} / \mathrm{mm}$ and $\sim 2.05 \mathrm{~dB} / \mathrm{mm}$, respectively, corresponding to an excess propagation loss of $\sim 1.63 \mathrm{~dB} / \mathrm{mm}$ induced by the GO film. This is over 1 order of magnitude lower than that of silicon waveguides coated with undoped graphene [33]. Figure 2(c) shows the power-dependent excess insertion loss (EIL, defined as the additional insertion loss over that in (a)) of the optical pulses (pulse width: $\sim 3.7 \mathrm{ps}$, repetition rate: $\sim 60 \mathrm{MHz}$ ) after going through the hybrid waveguides, which includes the loss induced by TPA of silicon waveguide and also the change of GO material properties.
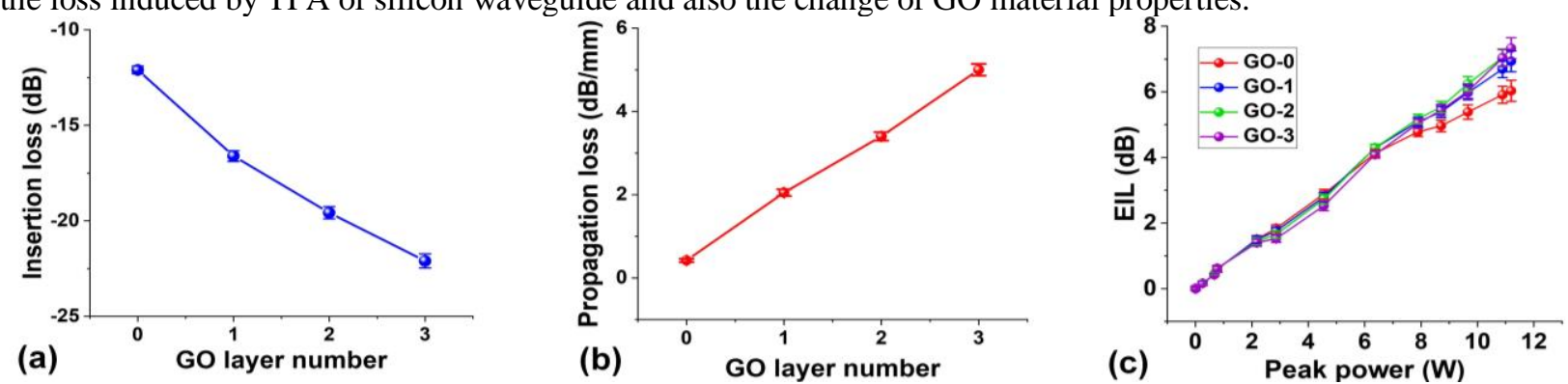

Fig. 2. (a) Insertion loss and (b) propagation loss of the GO-silicon hybrid waveguides with 1-3 layers of GO. (c) Power-dependent EIL of optical pulses after passing the hybrid waveguides. The results for the bare silicon waveguide (GO-0) are also shown for comparison.

\section{SPM in GO-silicon hybrid waveguides}

Figure 3(a) shows normalized optical spectra of the picosecond optical pulses after going through the hybrid waveguides, together with those of the input pulses and the pulses after passing the bare silicon waveguide. For comparison, we kept the same peak power of $11 \mathrm{~W}$. As compared with the bare silicon waveguide, there are obviously broadened spectra for all the hybrid waveguides. The asymmetric spectral broadening is mainly induced by the freecarrier effects of silicon. Figure 3(b) shows the calculated BF defined as the square of the pulses' RMS spectral width at the waveguide output facet divided by the corresponding value at the input $[34,35]$. There is a maximum $\mathrm{BF}$ of 2.96 for the waveguide with 2 layers of GO, reflecting the trade-off between SPM enhancement and loss increase. The optical spectra measured at different input peak powers for the waveguide with 2 layers of GO are shown in Fig. 3 (c). By fit the results with SPM theory [31, 35], we obtained a $n_{2}$ value of $\sim 2.68 \times 10^{-14} \mathrm{~m}^{2} / \mathrm{W}$ for 2 layers of GO, which is about 4 orders of magnitude higher than silicon and agrees with our previous FWM experiments [31].
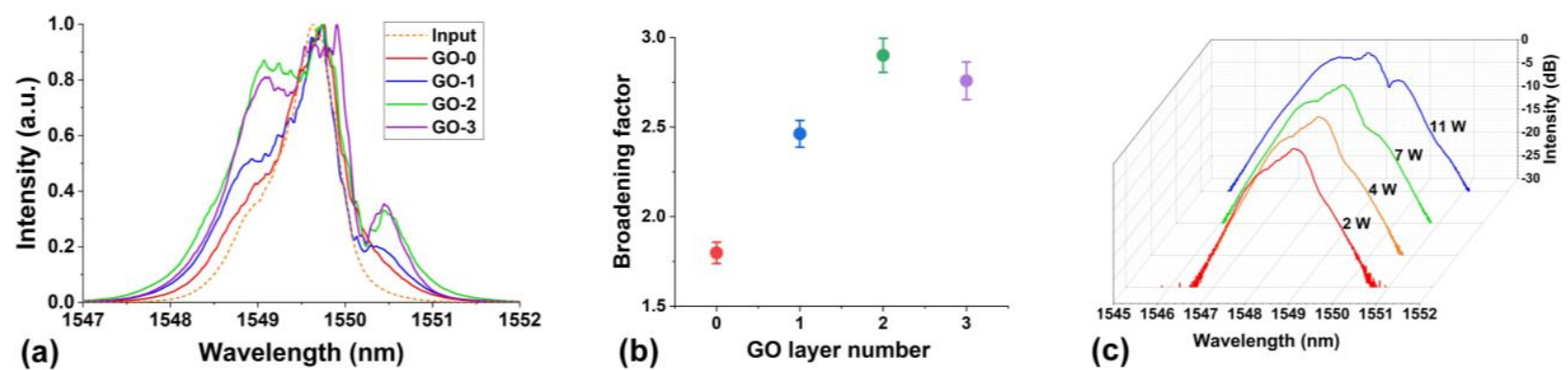

Fig. 3. (a) Normalized optical spectra of the picosecond optical pulses before and after going through the bare and hybrid waveguides. (b) Calculated BFs for the optical spectra in (a). (c) Optical spectra measured at different peak powers for the waveguide with 2 layers of GO.

\section{Conclusion}

We demonstrate enhanced SPM in silicon nanowire waveguides integrated with 2D layered GO films. We achieve significant spectral broadening of optical pulses in the GO-silicon waveguide with a high BF of 2.96.

\section{Reference}

[1] J. Leuthold, C. Koos, and W. Freude, "Nonlinear silicon photonics," Nat. Photon., vol. 4, no. 8, 535, 2010.

[2] J. Wu et al, "RF photonics: An optical micro-combs' perspective", IEEE J. Selected Topics in Quantum Electronics, vol. 24, no. 4, pp. 1-20, Article: 6101020. 2018. DOI: 10.1109/JSTQE.2018.2805814.

[3] B.J. Eggleton, S.Radic, and D.J. Moss, Chapter 20, p759-828 in Optical Fiber Telecommunications V: Components and Sub-systems, Edited by Ivan P. Kaminow, Tingye Li, and Alan E. Willner, Academic Press, Oxford, UK, February (2008).

[4] Li, F. et al., Optics Express, vol. 19, 20364-20371 (2011). DOI: 10.1364/OE.19.020364.

[5] Li, F. et al., Optics Express, vol. 18, 3905-3910 (2010). DOI: 10.1364/OE.18.003905.

[6] C.Monat et al., "Integrated optical auto-correlator based on third-harmonic generation in a silicon photonic crystal waveguide", Nature Communications, vol. 5 Article number: 3246, Feb 5, (2014) doi:10.1038/ncomms4246. 
[7] Monat, C. et al., Optics Express, vol. 18 (7), 6831-6840 (2010). DOI: 10.1364/OE.18.006831.

[8] Corcoran, B., et. al., Optics Express, vol. 18, (8) 7770-7781 (2010). DOI: 10.1364/OE.18.007770.

[9] D. J. Moss, H. M. van Driel, and J. E. Sipe, Opt. Lett., vol. 14, no. 1, p57 (1989).

[10] D. J. Moss, E. Ghahramani, J. E. Sipe, and H. M. van Driel, Phys. Rev. B, vol. 41, no. 3, 1542 (1990).

[11] D. J. Moss, H. M. van Driel, and J. E. Sipe, Appl. Phy. Lett., vol. 48, no. 17, 1150 (1986).

[12] D. J. Moss, et al., Electronics Letters, vol. 41, 320 (2005). DOI:10.1049/el:20058051

[13] M.R.E. Lamont, et al., IEEE Photonics Technology Letters, vol. 18, 1185 (2006). DOI:10.1109/LPT.2006.874718.

[14] A.Tuniz, G.Brawley, D.J.Moss, B.J.Eggleton, Optics Express, vol. 16, 18524 (2008). DOI: 10.1364/OE.16.018524

[15] MD Pelusi, F Luan, E Magi, MRE Lamont, DJ Moss, BJ Eggleton, "High bit rate all-optical signal processing in a fiber photonic wire", Optics Express, vol. 16, no. 15, 11506-11512 (2008).

[16] M.Lee, et al., Optics Express, vol. 15, pp. 1277-1285 (2007). DOI:10.1364/OE.15.001277

[17] S. Tomljenovic-Hanic, M.J. Steel, C. Martijn de Sterke and D. J. Moss, "High-Q cavities in photosensitive photonic crystals", Optics Letters, vol. 32, no. 5, 542-544 (2007). DOI:10.1364/OL.32.000542

[18] C. Grillet, et al., Optics Express, vol. 15, no. 3, 1267-1276 (2007). DOI:10.1364/OE.15.001267

[19] V.G. Ta'eed et al., "Chalcogenide Glass Photonic Crystal Devices", Photonic and Electromagnetic Crystal Structures, Photonics and Nanostructures-Fundamentals and Applications, vol. 6, no. 1, 3-11 (2008). DOI:10.1016/j.photonics.2007.11.001.

[22] V.G. Ta'eed et al., "Ultrafast all-optical chalcogenide glass photonic circuits", Optics Express, vol. 15, 9205 (2007).

[23] Y. Yang et al., "Enhanced four-wave mixing in waveguides integrated with graphene oxide," APL Photonics, vol. 3, no. 12, 120803. 2018. doi: $10.1063 / 1.5045509$.

[24] Y. Qu et al., "Enhanced four-wave-mixing and 3rd order optical nonlinearity in SiN nanowires integrated with graphene oxide films," arXiv:2006.14944, 2020.

[25] Y. Zhang et al., "Enhanced Kerr Nonlinearity and Nonlinear Figure of Merit in Silicon Nanowires Integrated with 2D Graphene Oxide Films," ACS Applied Materials and Interfaces, vol. 12, no. 29, 33094-33103, Jul 10. 2020. DOI:10.1021/acsami.0c07852

[26] J. Wu et al., "Graphene Oxide Waveguide and Micro-Ring Resonator Polarizers," Laser \& Photonics Reviews, vol. 13, no. 9, 1900056. 2019. DOI:10.1002/lpor.201900056.

[27] J. Wu et al., "2D Layered Graphene Oxide Films Integrated with Micro-Ring Resonators for Enhanced Nonlinear Optics," Small, vol. 16, no. 16, 1906563 April 23 (2020). DOI: 10.1002/smll.201906563.

[28] T. Gu et.al., "Regenerative oscillation and four-wave mixing in graphene optoelectronics," Nat. Photon., 6(8), 554, 2012.

[29] Y. Yang et.al., "Bottom-up fabrication of graphene on silicon/silica substrate via a facile soft-hard template approach," Sci. Rep., 5, 1, 2015.

[30] X. Zheng et.al., "In situ $3^{\text {rd }}$ non-linear responses during laser reduction of graphene oxide thin films towards on-chip non-linear photonic devices," Adv. Mater., 26(17), 2699, 2014

[31] Y. Yang et.al., "Enhanced four-wave mixing in waveguides integrated with graphene oxide," APL Photonics, 3 (12), $120803,2018$.

[32] J. Wu et.al., "Graphene oxide waveguide and micro-ring resonator polarizers," Laser Photonics Rev., 13(9), 1900056, 2019.

[33] R. Kou et.al., "Characterization of optical absorption and polarization dependence of single-layer graphene integrated on a silicon wire waveguide," Japanese Journal of Applied Physics, 52, 060203, 2013.

[34] N. Vermeulen et.al., "Graphene's nonlinear-optical physics revealed through exponentially growing self-phase modulation," Nat. Comm., 9(1), 2675, 2018.

[35] L. Yin et.al., "Impact of two-photon absorption on self-phase modulation in silicon waveguides," Opt. Lett., 32(14), $2031,2007$. 